\title{
Diagnostic of Capacitively Coupled Low Pressure Radio Frequency Plasma: An Approach through Electrical Discharge Characteristic
}

\author{
B. Bora, H. Bhuyan, M. Favre, E. Wyndham, and H. Chuaqui
}

\begin{abstract}
Low temperature radio frequency plasma is widely used in low temperature plasma processing medium for material processing in many fields including microelectronics, aerospace, and the biology. For proper utilization of the process, it is very much important to know the plasma parameters. In this paper a technique is reported to determine the plasma parameters from the electrical discharge characteristic of a capacitivly couple radio frequency argon plasma. The homogeneous discharge model is modified to make it applicable in low pressure by incorporating the plasma series resonance effect. The effect on the plasma resistance by the change in drift velocity of the electron with rf electric filed is also considered. The electron density and temperature is found to be well agreed with the Langmuir probe diagnostic result, which is in the range of $0.5 \times 10^{10}$ to $4.5 \times 10^{10} \mathrm{~cm}^{-3}$ and 1.4 to $1.6 \mathrm{ev}$ for wide range of $\mathrm{rf}$ power.
\end{abstract}

Index Terms-Capacitive couple radio frequency plasma, discharge characteristic, homogeneous discharge model, plasma parameters, power balance.

\section{INTRODUCTION}

Radio-frequency (rf) plasma is widely used as a low temperature plasma processing medium for material processing in many fields including microelectronics, aerospace, and the biology [1,2]. As a result of energetic ions, chemically active species, radicals and also energetic neutral species, rf discharges are widely used in etching, deposition and surface treatment, particularly in the semiconductor industry. Although, much processing is done on an empirical basis for a particular device, a full characterization is desirable for reproducibility, consistency, better understanding the process, and more importantly transformation of process from one device to another [3]. The yield of ion implantation process like plasma enhanced chemical vapor deposition (PECVD), plasma immersion ion implantation (PIII) strongly depends on the ion density [4]. It is therefore important to investigate the plasma parameters in such discharge. There are several diagnostic techniques employed for the determination of electron density and temperature in dc plasmas which includes Langmuir probe [5, 6], plasma spectroscopy [7,] microwave and laser interferometries, and Thomson scattering [8-10]. Several

Manuscript received September 5, 2011. The author B. Bora acknowledges CONICYT for funding the postdoctoral fellowship grant FONDECYT 3110008.

Department of Physics, Pontificia Universidad Catolica de Chile, Santiago, Chile. (e-mail: bbora@ fis.puc.cl). characterization techniques are reported in recent literature to characterize the atmospheric pressure capacitively couple radio frequency (CCRF) plasma for different experimental parameters [11-14]. The electrical discharge characteristic can be also used to estimate the plasma parameters, which is simpler, easier, quicker and no additional equipment is required [11]. Li et al [11] estimated the electron density and electron temperature from the current-voltage and current-power characteristic of an atmospheric pressure CCRF plasma by using homogeneous discharge model. They have estimated the plasma density in $\alpha$ mode of atmospheric pressure CCRF plasma. They assumed that the electron density $n_{e}$ is only proportional to the rms value of the applied current because of the frequent electron neutral collision in atmospheric pressure, which makes the drift velocity of the electron independent on the variation of applied electric field [11]. This model can be used to evaluate the plasma parameters in atmospheric pressure CCRF plasma. However, large range of measurements on discharge characteristics is required to evaluate the plasma parameters. In addition, in low pressure, which is very much important in material processing works when the electron neutral collision reduced and the electron gain more energy from the applied electric field, this model cannot be used directly to evaluate the plasma parameters. In addition, the nonlinear plasma series resonance (PSR) effect is also dominant in low pressure particularly in mtorr range, and produces several harmonics to the rf current, which need to be considered in calculating the plasma parameters.

In this paper, we have reported a method to evaluate the plasma parameters from the electrical discharge characteristic of CCRF plasma system in low pressure argon environment. The homogeneous discharge model of CCRF is modified to make it applicable in low pressure particularly in mtorr range by adopting the nonlinear PSR effect. In this method, we have also consider that the plasma density depends on the rms current as well as rms voltage, since the drift velocity of the electron is strongly affected by applied electric field in low pressure due to the reduction in collision. Furthermore this model is used to evaluate the plasma parameters from the experimental data for wide range of power and operational parameters.

\section{EXPERIMENTAL DETAILS}

The experiment is carried out in a $13.56 \mathrm{MHz}$ CCRF system. The experimental setup of the CCRF system is shown in Fig. 1. The electrode assembly of the rf discharge 
consists of two water cooled circular disc shaped parallel stainless steel electrodes separated by $11 \mathrm{~cm}$. The diameter of the lower powered electrode is $8 \mathrm{~cm}$ and the upper grounded electrode is $10 \mathrm{~cm}$. The pressure in the stainless steel vacuum chamber was maintained by a combination of rotary and turbo molecular pumps. Series of mass flow controller are used for

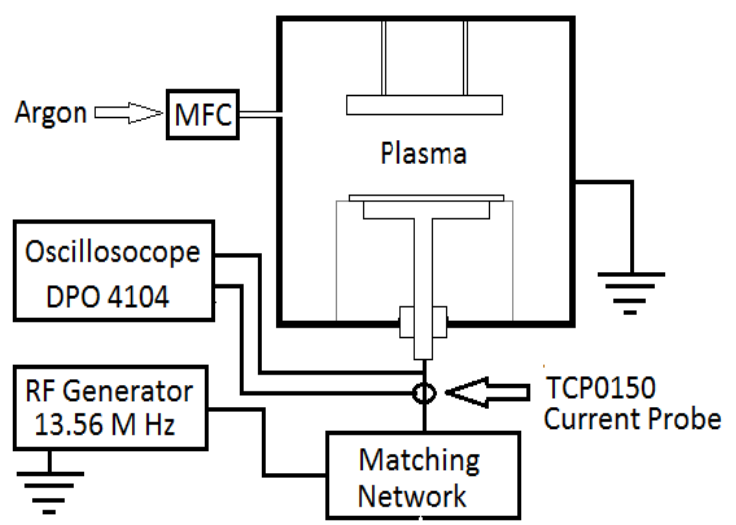

Fig. 1.Experimental setup.

maintaining gas flow rates. The discharge current is measured by a Tektronix TCP0150 current probe and the signals are recorded in a Tektronix DPO-4104 oscilloscope for further analysis. An automatic matching network (ENI MWH-5-01) is connected in between the rf power supply and the electrodes to deliver maximum power to the load. The rf power generator (ENI ACG-6B) can deliver up to a maximum of 600 watt and is equipped with a pair of meters to monitor simultaneously the forward power and reflected power. Electrical signals from the plasma are recorded only when there is no reflected power from the load. Bulk plasma is produced and filled in the space between the electrodes and the electrodes are covered by sheath regions. A negative dc potential is develops between the bulk plasma and the power electrodes, which is commonly known as 'self bias' in rf plasma. This self bias is generated as a consequence of the geometrical asymmetry of the electrode system. Due to this 'self bias' the ion energies near the power electrode reaches up to few hundred $\mathrm{eV}$. This feature makes the rf discharge suitable for application in different material processing areas $[2,15-19]$. The experiment is carried out in 35 mtorr chamber pressure and the rf power is varied from 10 watt to 120 watt. The three measured quantities i.e. $I_{r m s}, V_{r m s}$, and average power $P$ are used to calculate plasma parameters from the modified homogeneous discharge model as describe in the next section.

\section{The EQUATIONS AND Modeling}

In homogeneous discharge model of CCRF, the plasma is considered as a series of sheath capacitor $\left(C_{s}\right)$, a bulk plasma resistor $\left(R_{p}\right)$, and a bulk plasma inductor $\left(L_{p}\right)$ as shown in Fig. 2.

In the bulk of the discharge plasma, the electron frequency $\left(\omega_{p}\right)$, the capacitance of the parallel electrode $\left(C_{0}\right)$ can be used to express the inductance due to the inertia of the electron in the bulk of the plasma $[11,20]$,

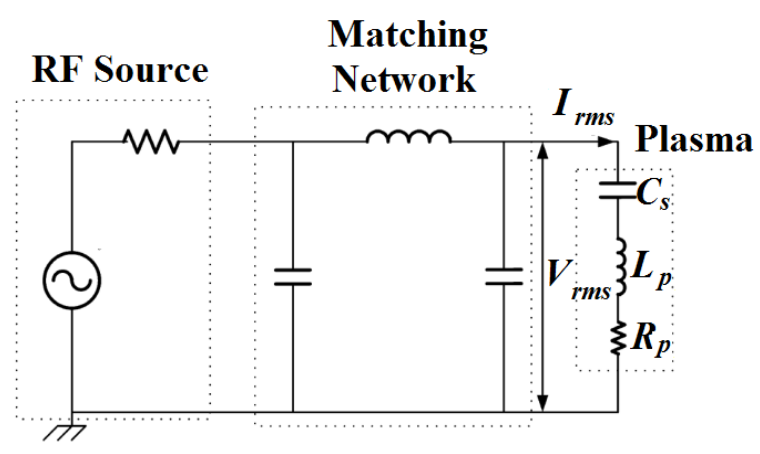

Fig. 2.Equivalent circuit of the CCRF plasma discharge according to homogeneous discharge model.

$L_{p}=\frac{1}{\omega_{p}^{2} C_{0}}=\frac{m d}{A n_{e} e^{2}}$

where, $\mathrm{m}$ is the mass of the electron, $d$ is the distance between the electrodes, $A$ is the surface area of the parallel plate, $n_{e}$ is the electron density. The plasma bulk is assumed to be quasineutral and (for simplicity) homogeneous. The radio frequency current through the discharge is carried by electron conduction alone. The dependence of the current density on the electric field can thus be modeled by a generalized Ohm's law. It takes into account the acceleration of the electrons by the electric field and their momentum loss due to elastic collisions with the neutrals of the background gas. The collision rate is given by momentum transfer collision frequency. For simplicity, considering only the Ohmic heating the resistance of the bulk plasma can be express as $[11,20]$

$R_{p}=\frac{v L_{p}}{c_{f}}$

where, $v$ is the electron neutral collision frequency. $c_{f}$ is a correction factor introduced to the plasma resistance to take into account the change in drift velocity of the electrons with the variation of the electric field and the PSR effect. Since the electrons have sufficient distance to travel and accelerate in the bulk plasma at low pressure, we anticipate that the resistance of the bulk plasma changes due to the change in drift velocity of the electrons. Besides, it was reported that due to the PSR effect the measured rms current is higher than the current predicted by homogeneous discharge model [13, 14]. It has also been experimentally observed that at the beginning of the rf cycle the plasma sheath expands and as a result energetic electron beams are generated [14, 21]. The PSR effect enhances the generation of these highly energetic electron beams due to the rapid expansion of the sheath. These electrons travel from the sheath region and propagate through the bulk plasma and enhance the total plasma current. The electron neutral collision frequency can be express as [20],

$v=n_{e} \pi d_{c o l}^{2}\left(\frac{8 k T_{e}}{\pi m}\right)^{\frac{1}{2}}$

where, $d_{c o l}$ is the electron neutral collision cross section and 
$T_{e}$ is the electron temperature. Combining equation (2) and (3),

$R_{p}=\frac{d d_{c o l} 2}{A e^{2} c_{f}}\left(8 \pi m k T_{e}\right)^{\frac{1}{2}}$

In homogenous model, the sheath capacitance can be express as $[11,20]$

$C_{s}=\frac{e n_{e} \omega \varepsilon_{0} A^{2}}{2 I_{r m s}}$

The rms values of current $\left(I_{r m s}\right)$ and voltage $\left(V_{r m s}\right)$ can be correlated according to the homogeneous discharge model and can be express as,

$$
V_{r m s}^{2}=I_{r m s}^{2}\left[\begin{array}{l}
8 \pi m k \frac{1}{c_{f}^{2}}\left(\frac{d d_{c o l}^{2}}{A e^{2}}\right)^{2} T_{e} \\
+\left(\frac{m d \omega}{A e^{2}}-\frac{2}{e \varepsilon_{0} \omega^{2} A^{2}} I_{r m s}\right)^{2} \frac{2}{n_{e}^{2}}
\end{array}\right]
$$

Shou-Zhe Li et al [11] shows that for atmospheric pressure CCRF plasma the rms value of voltage follows the variation of the rms value of current according to one branch of hyperbola. This is a special case of the equation (6), when the drift velocity of the electron in the bulk of plasma depends trivially with the variation of electric field due to the frequent electron-neutral collision at the atmospheric pressure.

The dissipated power of the plasma and the rms values of current and voltage can be express in terms of power factor as,

$$
P=V_{r m s} I_{r m s} \cos \phi
$$

where, $\phi$ is the phase difference. The equation (7) can be express from the equivalent circuit of the homogeneous discharge model as,

$$
\begin{aligned}
& \cos ^{-1}\left(\frac{P}{V_{r m s} I_{r m s}}\right)=\tan ^{-1} \\
& {\left[\frac{m \omega c_{f}}{(8 \pi m k)^{\frac{1}{2}} d_{c o l}^{2}}\left(1-\frac{2 e}{\omega^{3} \varepsilon_{0} A m d} I_{r m s}\right) \frac{1}{n_{e}} T_{e}^{\frac{1}{2}}\right]}
\end{aligned}
$$

The power density $\mathrm{p}(\mathrm{W} / \mathrm{cm} 3)$ can be express in terms of temperature and density as power balance equation [11],

$$
\begin{aligned}
& p=n_{e} n_{g} K_{i z} \varepsilon_{i z}+n_{e} n_{g} K_{e x} \varepsilon_{a^{*}}+n_{e} n_{a^{*}} K_{i z^{*}} \varepsilon_{i z^{*}} \\
& +n_{e}\left[\begin{array}{l}
n_{i} K_{e l, e-i}+n_{g} K_{e l, e-a} \\
+n_{a^{*}} K_{e l, e-a^{*}}
\end{array}\right] \frac{2 m_{e}}{M} \frac{3}{2}\left(T_{e}-T_{g}\right) \\
& +\varepsilon_{\text {rad }}
\end{aligned}
$$

where, $T_{g}$ is the gas temperature, $K_{i z}$ and $\varepsilon_{i z}$ are the ionization rate coefficient and first ionization energy from the ground state. $a^{*}$ represents the first excited state of the atom, $\varepsilon_{a}{ }^{*}$ and $\varepsilon_{i z}{ }^{*}$ are the first excitation energy and ionization energy of the state $a^{*}, K_{e x}$ is the excitation rate coefficients, and $K_{i Z^{*}}$ is the ionization rate coefficient from the first excited state. In this equation, $K_{e l, e-i}, K_{e l, e-a}$, and $K_{e l, e-a *}$ are the electron-ion, electron-atom, and electron- excited state collision rate coefficients respectively. Eq. (9) includes many energy dissipation factors involved in the discharge process; however, in the case of cold plasma generation with low power density, many terms becomes negligible. The radiation loss does not influence considerably on the power balance equation for the cold non-equilibrium plasmas. Hence, considering only the significant terms in Eq. (9), the power balance equation reduce to,

$p=n_{e} n_{g} K_{i z} \varepsilon_{i z}+n_{e} n_{g} K_{e x} \varepsilon_{a^{*}}+n_{e} n_{g} K_{e l, e-a} \frac{3 m}{M} T_{e}$

The temperature dependent terms in equation (10) can be express as [11, 12],

$K_{i z}^{A r}\left(T_{e}\right)=4 \times 10^{-8} T_{e}^{0.5} \exp \left(-15.8 / T_{e}\right)$

$K_{e x}^{A r}\left(T_{e}\right)=1 \times 10^{-9} T_{e}^{0.5} \exp \left(-11.6 / T_{e}\right)$

$K_{e i, e-a}^{A r}\left(T_{e}\right)=\left(0.084+0.537 T_{e}+1.192 T_{e}^{2}\right) \times 10^{-8}$

The equation (6), (8), and (10) are solve simultaneously coupling with the equation (11-13) to determine the three unknown plasma temperature $T_{e}$, plasma density $n_{e}$ and the correction factor $c_{f}$

\section{RESUlt AND DiscUSSION}

The instantaneous plasma current for different operational power are shown in Fig. 3 at 35 mtorr chamber pressure. From this figure it is observed that the phase difference of the

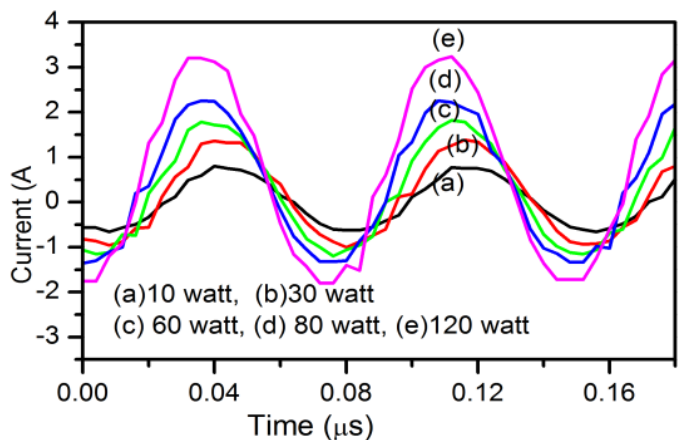

Fig. 3.Typical current waveforms at $35 \mathrm{mtorr}$ pressure for different rf power current is varying with different rf power. The distortion observed in the current waveforms is a clear indication of the PSR effect. The PSR effect is observed for all the current waveforms irrespective of rf power. To study the PSR effect, 
Fast Fourier Transformation (FFT) of the current waveforms is performed and the results are shown in the Fig. 4. Several harmonics of the applied frequency are observed in the

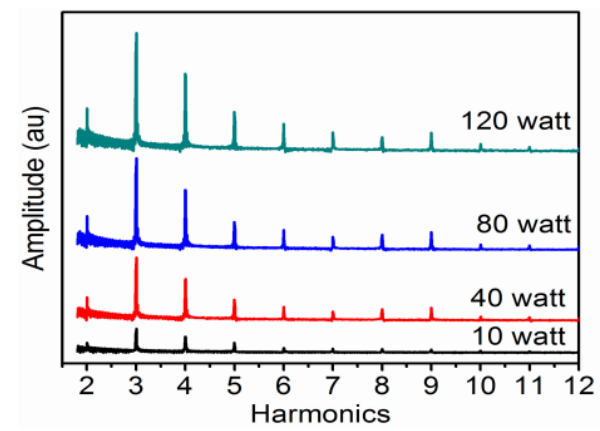

Fig. 4.Fast Fourier Transform of the current waveforms for different rf power at $35 \mathrm{mtorr}$ argon operating pressure.

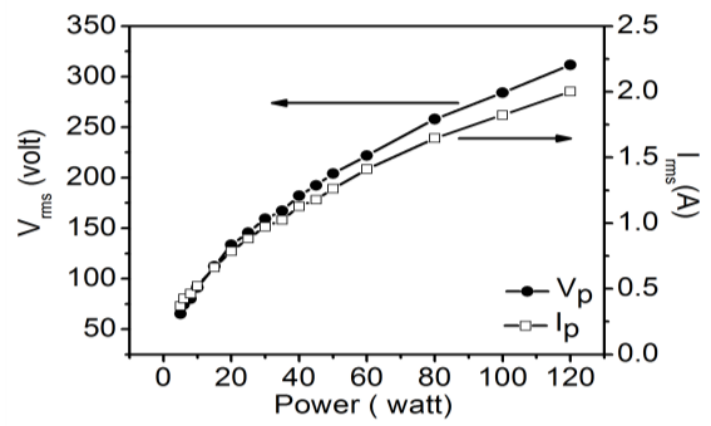

Fig. 5.Variation of measured $V_{r m s}$ and $I_{r m s}$ with operational rf power

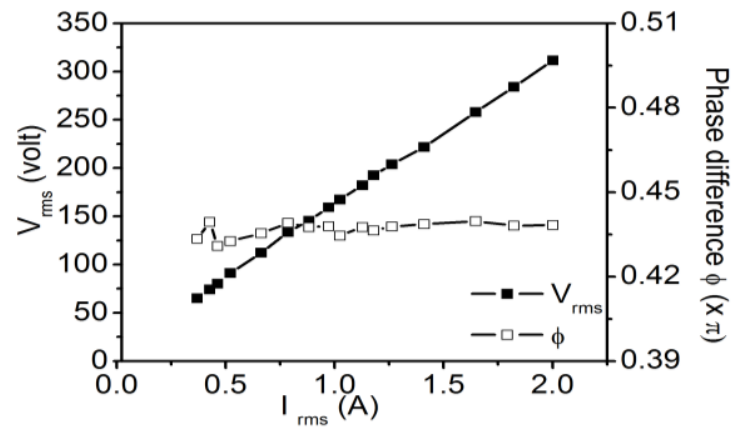

Fig. 6. $V_{r m s}-I_{r m s}$ characteristic and the change in the phase difference with $I_{r m s}$.

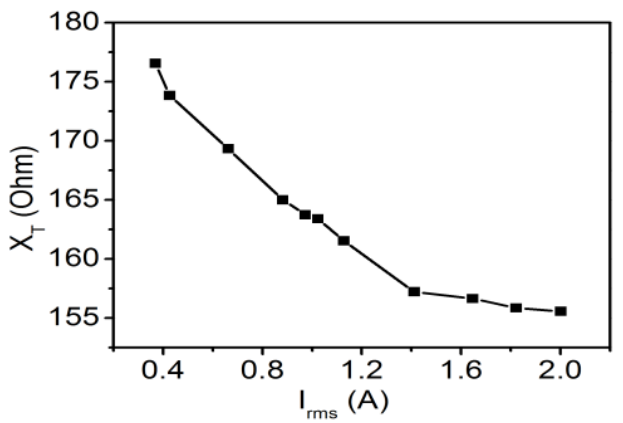

Fig. 7.Variation of total plasma impedance with $I_{r m s}$.

current waveforms. The intensities of the harmonics are seen to increase with increasing rf power with a maximum intensity of the third harmonic. The value of the $V_{r m s}$ is calculated from the voltage waveforms after subtracting the DC components produced by self-bias [22]. The variation of the measured $I_{r m s}$ and $V_{r m s}$ with rf power are shown in Fig. 5. Both the $V_{r m s}$ and $I_{r m s}$ are seen to increase with increasing rf power. The corresponding current density calculated to be in the range of $4.7 \mathrm{~mA} / \mathrm{cm}^{-2}$ to $25.5 \mathrm{~mA} / \mathrm{cm}^{-2}$. During the experiment it is observed that the plasma is completely filled in between the electrode. The $V_{r m s}-I_{r m s}$ characteristic and the phase difference between the current and voltage are shown in Fig. 6. The $V_{r m s}-I_{r m s}$ characteristic is almost linear in our present experimental range and it is almost similar with the previously reported characteristic obtained in atmospheric pressure rf plasma $[11,23,24]$ for higher current density, which can be assumed as a straight line. However, the $V_{r m s}$ $I_{r m s}$ characteristic at lower current density is different from the atmospheric pressure rf discharge $[11,23,24]$. It may be due to the considerable variation in resistance of the bulk plasma with the electric field in lower pressure and the existence of the PSR effect. The phase differences are observed to be in between $0.43 \pi$ to $0.44 \pi$, which is a direct consequence of the capacitively couple discharge. The variation of the total plasma impedance $\left(X_{T}=V_{r m s} / I_{r m s}\right)$ with $I_{r m s}$ is presented in Fig. 7. The $X_{T}$ is found to decreases with the increases $I_{r m s}$.

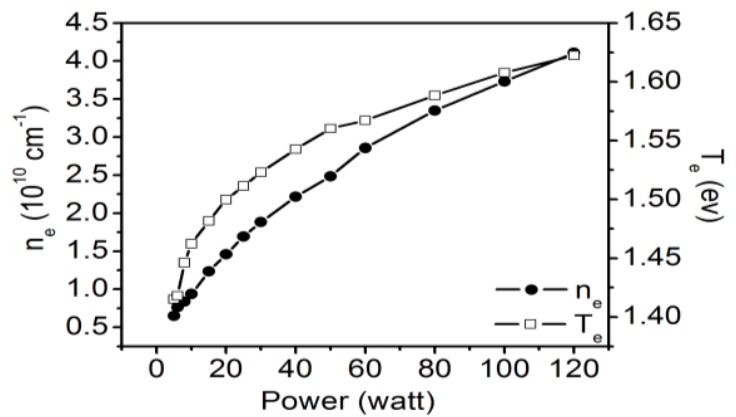

Fig. 8.Variation of calculated electron density and electron temperature with rf power.

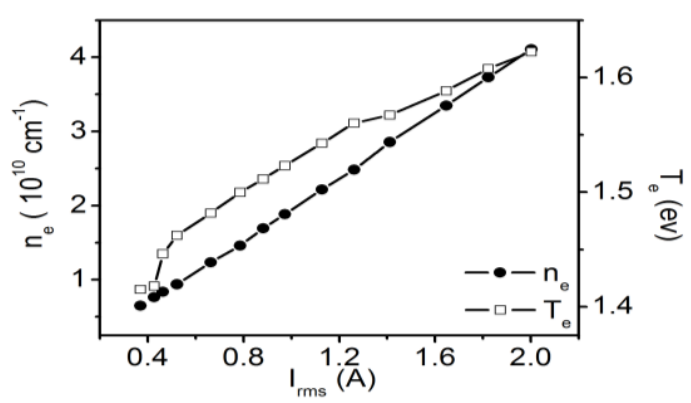

Fig. 9.Variation of calculated electron density and electron temperature with $I_{r m s}$.

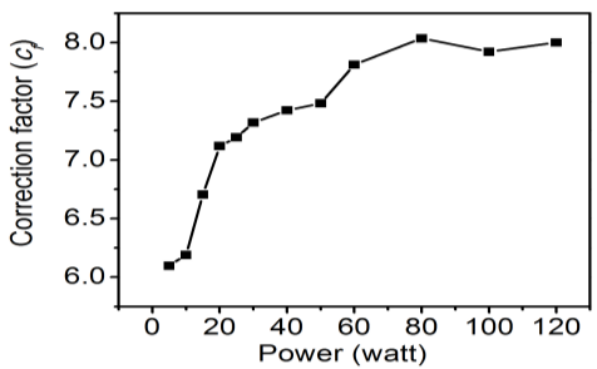

Fig. 10.Variation of calculated correction factor with $\mathrm{rf}$ power.

The computational results obtain for electron density and electron temperature for different rf power by solving the equation (6), (8), and (10-13) are shown in Fig. 8. The electron density are found to be in the range of $0.5 \times 10^{10} \mathrm{~cm}^{-3}$ to $4 \times 10^{10} \mathrm{~cm}^{-3}$, and the electron temperature in between 1.4 ev to $1.63 \mathrm{ev}$, which is well agree with the reported $\mathrm{rf}$ compensated Langmuir probe measurement data with the same experimental conditions [2]. The variation of electron density and electron temperature with $I_{r m s}$ are shown in Fig. 9, 
which shows a linear increment of density with current, which is obvious due to the increment of the collision with increasing current. The electron temperature is initially increased rapidly with increasing current and slow down the rate of increment as the current increased. It may be due to the increased of drift velocity of the electron with increasing electric field. Even the electric field is almost linearly increased with current, the drift velocity is not increasing linearly due to the increased of collision in higher current. This may be the cause for the nonlinear increased of electron temperature with $I_{r m s}$. The computed correction factor for different $\mathrm{rf}$ power is presented in Fig. 10. The correction factor is increased with increasing power, which is due to the increased of the drift velocity of the electron with increasing power. However, the observed correction factor is almost constant as the power increased beyond 80 watt. This is because the limitation on the increased in drift velocity with electric field at higher power due to collision. The correction factor is expected to become one in atmospheric pressure, when the drift velocity of the electron depends trivially with the variation of electric field due to the higher collision in atmospheric pressure and the PSR effect diminished completely. To find out the dependency of electron density on rf current a plot of $\alpha\left(=n_{e} / I_{r m s}\right)$ verses $I_{r m s}$ is presented in figure 11. Unlike the atmospheric pressure rf discharge where the electron density only depends linearly on current ( $\alpha=$ constant) [11], in low pressure the electron density shows considerably nonlinear dependency with current. The dependency factor in low pressure initially increases with increasing current and the rate of increment is reduces as the current increases further. These observations agreed with our initial assumption on the change in the plasma resistance due to the change in drift velocity of the electrons.

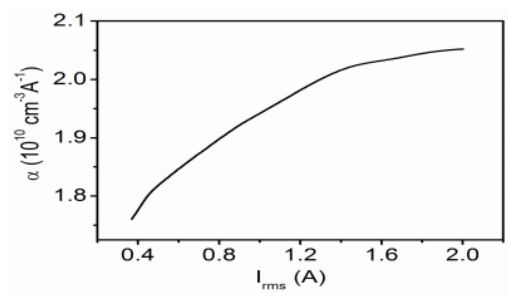

Fig. 11 .Dependency of electron density with $I_{r m s}$.

\section{CONCLUSION}

A simple computational model is developed to characterize the rf argon plasma from discharge characteristic and power. The homogeneous discharge model of CCRF discharge is modified by incorporating the PSR effect and extent it to evaluate the plasma parameters from the electrical discharge characteristic. This evaluated plasma parameters in a CCRF argon plasma in different rf power and the results arewell agreed with the results obtained by rf compensated Langmuir probe. This model can be also used in atmospheric pressure CCRF plasma.

\section{REFERENCES}

[1] K. H. Becker, U. Kogelschatz, K. H. Schoenbach, R. J. Barker, NonEquilibrium Air Plasmas at Atmospheric Pressure, Institute of Physics Publishing, Bristol, Philadelphia, 2005.
[2] E. Valderrama, M. Favre, H. Bhuyan, H.M. Ruiz, E. Wyndham, J. Valenzuela, H. Chuaqui, "Sub-micron size carbon structures synthesized using plasma enhanced CVD, without external heating and no catalyzer action", Surface \& Coatings Technology, vol. 204, pp. 2940-2943, 2010.

[3] A. Dyson, P. Bryant and J. E. Allen," Multiple harmonic compensation of Langmuir probes in rf discharges", Meas. Sci. Technol., vol. 11, pp 554-559, May 2000.

[4] S. M. Chen, R.M. Gwilliam and B.J. Sealy, "Computer simulation of plasma immersion ion implantation", Radiation Effects and Defects in Solids, vol. 141, pp. 149-159, 1997.

[5] R. H. Huddlestone and S. L. Leonard, Plasma Diagnostic Techniques Academic, London, 1965.

[6] B. Bora, M. Kakati, A.K. Das, "Variation of axial and radial temperaturein an expanded thermal plasma jet", J. Plasma Physics, vol. 76, pp. 699-707, 2010.

[7] H. R. Griem, Plasma Spectroscopy McGraw-Hill, New York, 1964.

[8] M. A. Heald and C. B. Wharton, Plasma Diagnostic with Microwaves Wiley, New York, 1965.

[9] M. C. M. Van de Sanden, J. M. de Regt, G. M. Janssen, J. A. M. Van der Mullen, D. C. Schram, and B. Van der Sijde, "A combined Thomson-Rayleigh scattering diagnostic using an intensified photodiode array", Rev. Sci. Instrum., vol 63, pp. 3369-3377, 1992.

[10] S. B. Cameron, M. D. Tracy, and J. P. Camaco, "Electron density and temperature contour plots from a laser-produced plasma using collective ultraviolet Thomson scattering”, IEEE Trans. Plasma Sci., vol. 24, pp 45-46. 1996.

[11] Shou-Zhe Li, Jin-Pyo Lim, Han S. Uhm, "Discharge characteristics of an atmospheric-pressure capacitively coupled radio-frequency argon plasmas", Physics Letters A, vol. 360, pp.304-308, 2006.

[12] M. A. Lieberman , A. J. Lichtenberg, E. Kawamura, T. Mussenbrock, and R. P. Brinkmann, "The effects of nonlinear series resonance on Ohmic and stochastic heating in capacitive discharges" Phys. Plasmas, vol. 15, pp. 063505, 2008.

[13] T. Mussenbrock, R. P. Brinkmann, M. A. Lieberman, A. J. Lichtenberg, and E. Kawamura, "Enhancement of Ohmic and Stochastic Heating by Resonance Effects in Capacitive Radio Frequency Discharges: A Theoretical Approach", Phys. Rev. Lett., vol. 101, pp. 085004, 2008.

[14] J. Schulze, B. G. Heil, D. Luggenholscher, T. Mussenbrock, R. P. Brinkmann, and U. Czarnetzki, "Electron beams in asymmetric capacitively coupled radio frequency discharges at low pressures", J. Phys. D: Appl. Phys., vol. 41, pp. 42003, 2008.

[15] C. Diplasu, A. Surmeian, A. Groza, and M. Ganciu, "Synchronous plasma enhancement in RF-driven plasma source for ion implantation", Surf. Coat. Technol., vol. 203, pp. 2858, 2009

[16] M. Jubault, L. Ribeaucourt, E. Chassaing, G. Renou, D. Lincot, and F. Donsanti, "Optimization of molybdenum thin films for electrodeposited CIGS solar cells", Sol. Energ. Mater. Sol. Cells, vol. 95, pp. S26, 2011.

[17] M.K. Lei, Y. Liu, and Y. P. Li., Appl. Surf. Sci., "Controllable wettability of poly(ethylene terephthlate) film modified by oxygen combined inductively and capacitively coupled radio-frequency plasma", vol. 257, pp.7350, 2011.

[18] J. W. Lee, H. S. Noh, S. H. Lee, J. H. Park, K. H. Choi, and S. J. Pearton, "Dry etching process of GaAs in capacitively coupled BCl3-based plasmas", Thin Solid Films, vol. 518, pp. 6488, 2010.

[19] C. M. Lin, W. C. Lien, V. V. Felmetsger, M. A. Hopcroft, D. G. Senesky, and A. P. Pisano, "AlN thin films grown on epitaxial $3 \mathrm{C}-\mathrm{SiC}$ (100) for piezoelectric resonant devices”, Appl. Phys. Lett., vol. 97, pp. 141907, 2010.

[20] M.A. Liebermann, A.J. Lichtenberg, Principles of Plasma Discharges and Materials Processing, Wiley, New York, 1994.

[21] Z. Donkó, J. Schulze, U. Czarnetzki, and D. Luggenhölscher, "Self-excited nonlinear plasma series resonance oscillations in geometrically symmetric capacitively coupled radio frequency discharges", Appl. Phys. Lett., vol. 94, pp. 131501, 2009.

[22] Annemie Bogaerts, Erik Neyts, Renaat Gijbels, Joost van der Mullen, "Gas discharge plasmas and their applications", Spectrochimica Acta Part B, vol. 57, pp. 609-658, April 2002.

[23] Yuan Tao Zhang, Qing Quan Li, Jie Lou, and Qing Min Li, "The characteristics of atmospheric radio frequency discharges with frequency increasing at a constant power density", Applied Physics Letters, vol. 97, pp. 141504-141506, 2010.

[24] J. L. Walsh, F. Iza, and M. G. Kong, "Atmospheric glow discharges from the high-frequency to very high-frequency bands", Applied Physics Letters, vol. 93, pp. 251502-251504, 2008. 